\title{
Sarcomatoid carcinoma of the pancreas: A case report and review of the literature
}

\author{
YINGJUN XIE*, YIEN XIANG*, DAN ZHANG, XIAOXIAO YAO, JIYAO SHENG, \\ YONGSHENG YANG and XUEWEN ZHANG
}

\begin{abstract}
Department of Hepatobiliary and Pancreatic Surgery, The Second Hospital of Jilin University, Changchun, Jilin 130041, P.R. China
\end{abstract}

Received March 10, 2018; Accepted September 7, 2018

DOI: $10.3892 / \mathrm{mmr} .2018 .9489$

\begin{abstract}
Sarcomatoid carcinoma (SC) is an extremely rare and complicated malignant neoplasm that consists of both malignant epithelial components and atypical spindle cells that express an epithelial phenotype. The presents study reported a case of SC of the pancreas (SCP), along with a brief review of the literature. A 63-year-old man was admitted to The Second Hospital of Jilin University hospital with complaints of epigastralgia and jaundice of one month in duration. Based on preoperative laboratory blood tests and radiography, a mass at the distal common bile duct was suspected Intraoperative examination discovered a $2.5 \times 2 \times 1.8-\mathrm{cm}$ mass in the pancreatic head, with invasion of the distal bile duct. Pancreaticoduodectomy was performed. Histopathology and immunohistochemistry of the specimen confirmed the diagnosis of SCP. The patient succumbed 18 months after surgery due to multiple hepatic metastases.
\end{abstract}

Correspondence to: Professor Yongsheng Yang or Professor Xuewen Zhang, Department of Hepatobiliary and Pancreatic Surgery, The Second Hospital of Jilin University, 218 Ziqiang Street, Changchun, Jilin 130041, P.R. China

E-mail: ysyang@jlu.edu.cn

E-mail: zhangxw@jlu.edu.cn

${ }^{*}$ Contributed equally

Abbreviations: SC, sarcomatoid carcinoma; CS, carcinosarcoma; SCP, sarcomatoid carcinoma of the pancreas; PD, pancreaticoduodenectomy; $\mathrm{CT}$, computed tomography; $\mathrm{CBD}$, common bile duct; MRCP, magnetic resonance cholangiopancreatography; TGF $\beta$, transforming growth factor beta; US, ultrasonography; EUS, endoscopic ultrasonography; MRI, magnetic resonance imaging; PTC, percutaneous transhepatic cholangiography; ERCP, endoscopic retrograde cholangiopancreatography; PPPD, pylorus-preserving PD

Key words: sarcomatoid carcinoma, pancreas, pancreaticoduodenectomy, spindle cells

\section{Introduction}

Sarcomatoid carcinoma (SC) or carcinosarcoma (CS) is a rare and complicated malignant neoplasm that consists of both malignant epithelial components and atypical spindle cells that express an epithelial phenotype $(1,2)$. SC or CS primarily occurs in the lungs, esophagus, breast, larynx, and gallbladder (3). SC of the pancreas (SCP) is extremely rare. Its clinical presentation is similar to that of pancreatic ductal adenocarcinoma. In most cases, the diagnosis is made on histopathological examination of the resected specimen. SCP is associated with poor prognosis. Surgery remains the mainstay of treatment. Due to rarity of the disease, no specific adjuvant therapy is available. Here, we report a patient with obstructive jaundice who was suspected to have distal cholangiocarcinoma and underwent pancreaticoduodenectomy (PD); the SCP diagnosis was made according to a detailed pathological examination.

\section{Case report}

A 63-year-old man was admitted to our hospital with the chief complaints of epigastralgia and jaundice of one month's duration. Significant past history included a weight loss of $10 \mathrm{~kg}$ in two months and a left nephrectomy due to left renal cancer one year before. Physical examination showed tenderness in the epigastrium. Laboratory blood tests revealed elevated serum liver enzymes levels, including alkaline phosphatase and $\gamma$-glutamyl transferase levels. The level of total serum bilirubin was $244 \mu \mathrm{mol} / 1$ (normal range: 5-21 $\mu \mathrm{mol} / \mathrm{l}$ ), with a direct serum bilirubin of $140 \mu \mathrm{mol} / 1$. Tumor marker levels were within normal ranges with the exception of mildly elevated CA72-4 levels $(10.38 \mathrm{U} / \mathrm{ml}$, normal range: $0.2-6.9 \mathrm{U} / \mathrm{ml})$. Abdominal computed tomography (CT) showed gross dilation of the bile ducts and gallbladder (Fig. 1A) with abrupt narrowing at the distal common bile duct (CBD) (Fig. 1B). Magnetic resonance cholangiopancreatography (MRCP) confirmed the above CT findings (Fig. 1C and D) and showed a beaklike change in the distal CBD (Fig. 1E and F). However, no definite mass lesion was visible. Based on the clinicoradiological findings, distal bile duct cancer was suspected, and the patient was prepared for surgery. Informed written consent was taken from the patient. Because there was no cholangitis, preoperative biliary drainage was not performed. During the surgery, a mass in 
the pancreatic head, measuring $2.5 \times 2 \times 1.8 \mathrm{~cm}$, was detected without any vascular invasion, and a PD was therefore performed.

Gross examination of the specimen showed a grayish white mass with variegated areas of necrosis and invasion of the distal CBD. Under the microscope, the cut specimen primarily consisted of staggered spindle cells with apparent atypia and frequent mitotic activities (Fig. 2A-C). Pleomorphic giant cells were also observed amid the spindle cells (Fig. 2A-C). However, we also observed some malignant epithelial components, and local invasion of the peripheral nerves. The lymph nodes, blood vessels and resection margins were free from tumor tissue. Immunohistochemistry showed that the spindle cells were positive for vimentin (Fig. 2D), CK7 (Fig. 2E) and CK19 (Fig. 2F). Thus, a diagnosis of SCP was confirmed.

The patient received thymopeptides ( $1 \mathrm{mg}$ per day) which is extracted from the traditional Chinese medicine for 15 days after surgery, to enhance immunity. His postoperative course was uneventful, and he was discharged from the hospital 10 days after surgery. At 16 months after surgery, the patient was found to have multiple hepatic metastases (Fig. 3). Because the patient's general condition was poor, no chemoradiation was offered. Palliative care and thymopeptides (1 mg twice per week) were given. He died 18 months after surgery.

\section{Discussion}

SCP is an exocrine neoplasm that originates from pancreatic ducts and acini (4). The most recent WHO classification of exocrine pancreatic tumors categorizes spindle cell carcinoma, SC and CS under a common heading of undifferentiated (anaplastic) carcinoma (5) because the spindle cells commonly express an epithelial immunohistochemical phenotype and/or genetic alterations in pancreatic ductal adenocarcinoma (6). Although several histogenetic mechanisms have been suggested for SC or CS, the exact mechanism is unclear. One proposed mechanism is that the mesenchymal components of $\mathrm{SC}$ include metaplasia from carcinoma under the influence of transforming growth factor beta (TGF $\beta)(3,7)$. CS formation is also suggested to occur when a monoclonal stem cell differentiates in two different directions (epithelial and mesenchymal components) under the stimulation of oncogenic factors (3).

SCP is commonly observed in older men with the average age of 60 years and its incidence is twice as high in males as in females according to previous data $(4,8,9)$. We collected and analyzed data from 23 patients with SC or CS of the pancreas (Table I) and found that the average age at diagnosis is $63.30 \pm 14.61$ years old. However, incidence rates between males and females are almost the same (10 male patients and 13 female patients). The most common symptoms of SCP include epigastralgia, poor appetite, abdominal distension, indigestion, diarrhea and weight loss. Vomiting and hematochezia may occur when the tumor invades the duodenum. Jaundice develops when the tumor infiltrates and obstructs the common bile duct. Tenderness in the epigastrium, with or without a mass, may be found via abdominal palpation. In the present case, the patient had obvious jaundice because of the partial displacement and obstruction of the distal CBD by the tumor.

Many imaging examinations are helpful for the diagnosis of SCP. Ultrasonography (US) is a convenient, noninvasive method for the initial screening of pancreatic tumors. SCP shows a low or mixed echo with a well-defined margin under US. Some indirect signs, such as the dilation of the common bile duct, may also be seen. However, disturbance of gas in the stomach and intestines limits US in diagnosing SCP. Endoscopic ultrasonography (EUS) overcomes this defect of US, with higher imaging resolution and diagnostic accuracy. Fine needle aspiration may also be performed during EUS, for biopsies, which are crucial for the preoperational diagnosis of SCP.

CT and enhanced CT are also widely used. SCP shows several manifestations on CT images. First, SCP primarily occurs in the pancreatic head and tail with an average diameter of $7.20 \pm 5.44 \mathrm{~cm}$, according to our data (pancreatic head: $n=13$, tail: $n=9$, body: $n=2$; papilla: $n=1$; Table I). Pancreatic head SCP can cause slight to moderate obstruction of the bile and pancreatic ducts. No atrophy of the pancreatic tail occurs. Second, nonenhanced CT images show well-circumscribed, hypodense or heterogeneous masses with cystic or solid lesions (10). Third, necrosis often occurs due to insufficient vessels and a solid-cystic mixed SCP structure (11). Fourth, small nodules of calcification may be observed in SCP. Fifth, enhanced CT images may show heterogeneous enhancement in the peripheral solid part of SCP, while the internal unilocular cystic part is often not enhanced (10). Last, due to tumor invasion, the peripancreatic lymph nodes, splenic artery, adjacent duodenum, liver and colon may be displaced and partly or entirely destroyed. In the present case, the small mass (which was invading the bile duct) was not clearly shown by radiography; however, the dilation of the bile ducts and gallbladder provided indirect evidence for the tumor. In magnetic resonance imaging (MRI), SCP shows abnormal signals, such as high or low signals in T2 weighted imaging $(12,13)$. Enhanced MRI is valuable for estimating the extent of local malignant involvement. Together with CT, MRI can clearly increase the diagnostic rate of pancreatic neoplasms, while preventing unnecessary injury during surgery by providing comprehensive imaging. Percutaneous transhepatic cholangiography (PTC) can clearly display the degree of bile duct dilation, thus facilitating diagnosis of pancreatic-head neoplasms. However, this invasive examination could be replaced by a noninvasive technique: MRCP, which provides a comprehensive display of the bile and pancreatic ducts at different levels. In the present case, dilation of the bile and pancreatic ducts indirectly indicated the existence of a periampullary tumor. Endoscopic retrograde cholangiopancreatography (ERCP) not only provides clear observation of periampullary neoplasms under direct vision, but also shows the dilation extent of the pancreatic and bile ducts through injection of contrast medium. Furthermore, tumors can be biopsied after papillotomy, thus providing important pre-surgical evidence for the diagnosis of SCP.

Histologically, SC consists of both malignant epithelial and mesenchymal components $(14,15)$, primarily mesenchymal components. The epithelial components can be adenocarcinoma or squamous cell carcinoma. According to our data (Table I), adenocarcinoma is the most common type (poorly differentiated: $n=7$, moderately differentiated: $n=8$, well-differentiated: $n=2$ ). Intersecting bundles of spindle cells with apparent atypia and frequent mitotic activities 

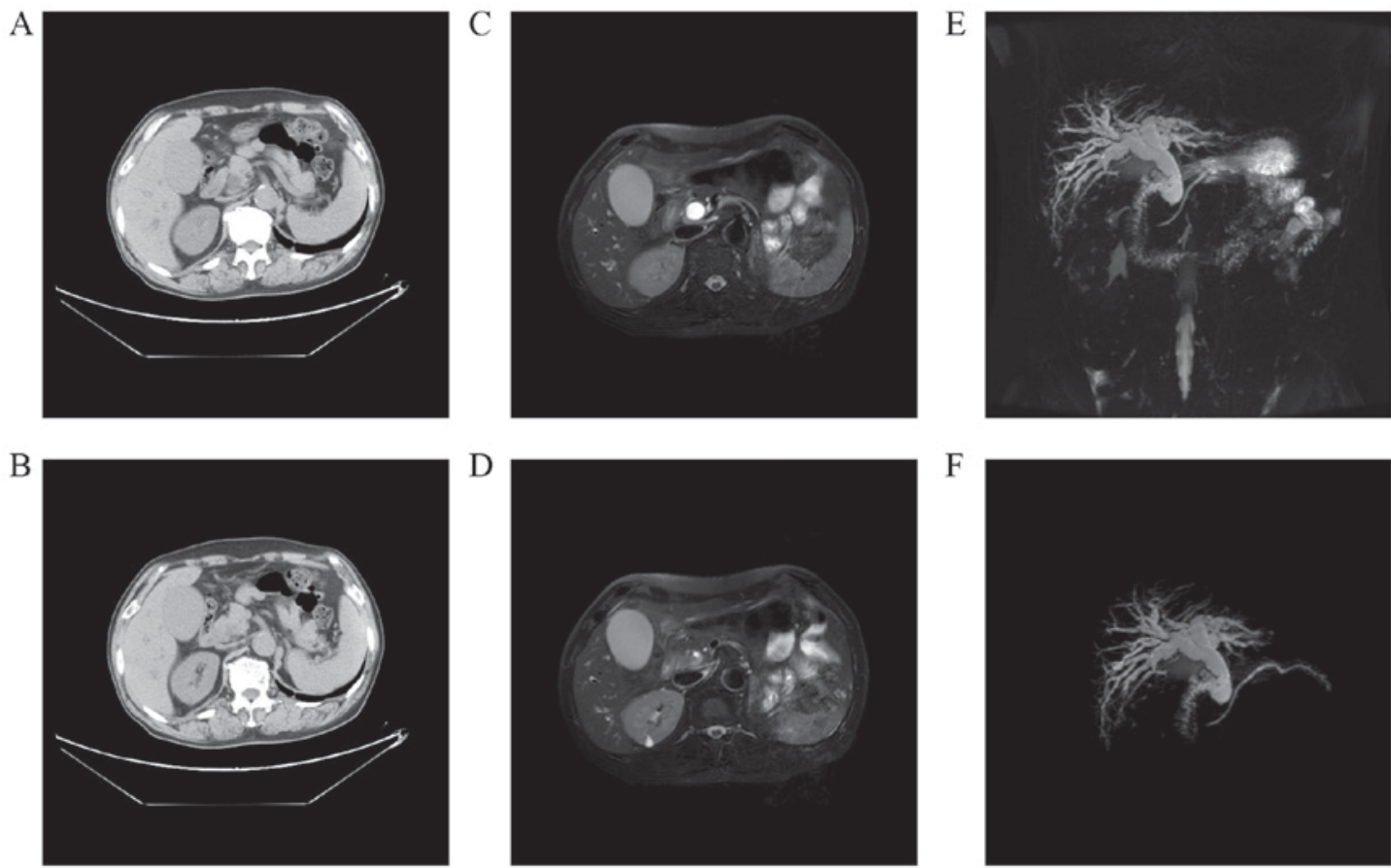

Figure 1. Gross dilatation of the bile ducts and gallbladder with abrupt narrowing and a beaklike change at the distal end of the common bile duct (CBD): (A and B) CT; (C-F) MRCP.
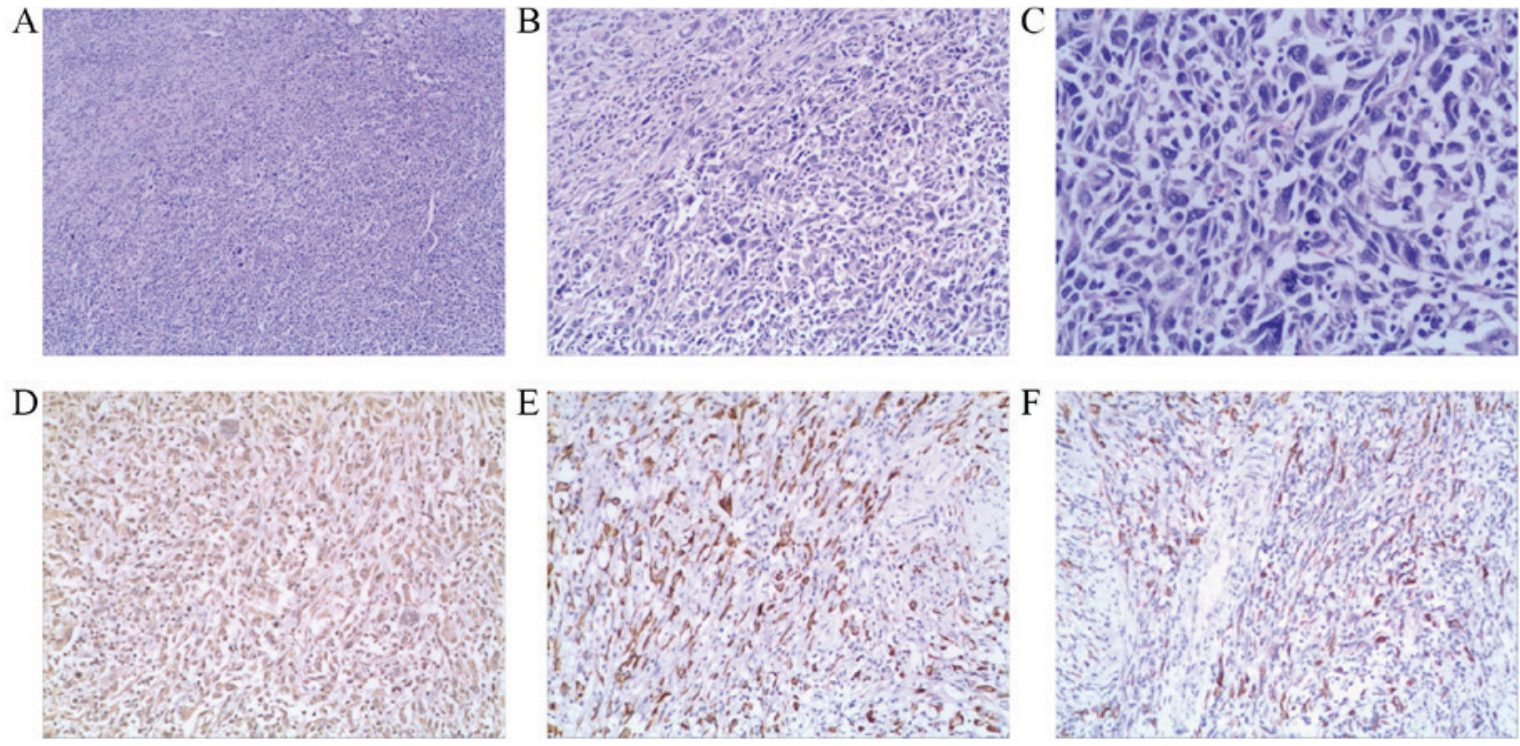

Figure 2. Histopathology of the resected specimen: Spindle cells with apparent atypia and frequent mitotic figures and occasional pleomorphic giant cells: (A) HE x40, (B) HE x100, (C) HE x200, (D) vimentin x100, (E) CK7 x100, (F) CK19 x100.
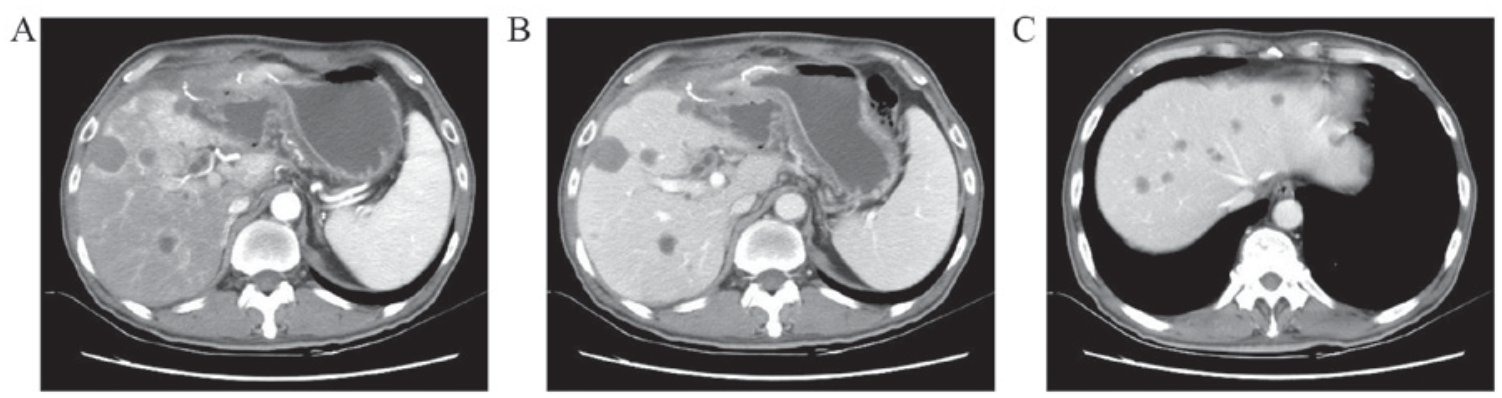

Figure 3. Contrast enhanced computed tomography showing multiple hepatic metastases appearing as hypodense lesions in (A) the arterial and (B and C) venous phase. 


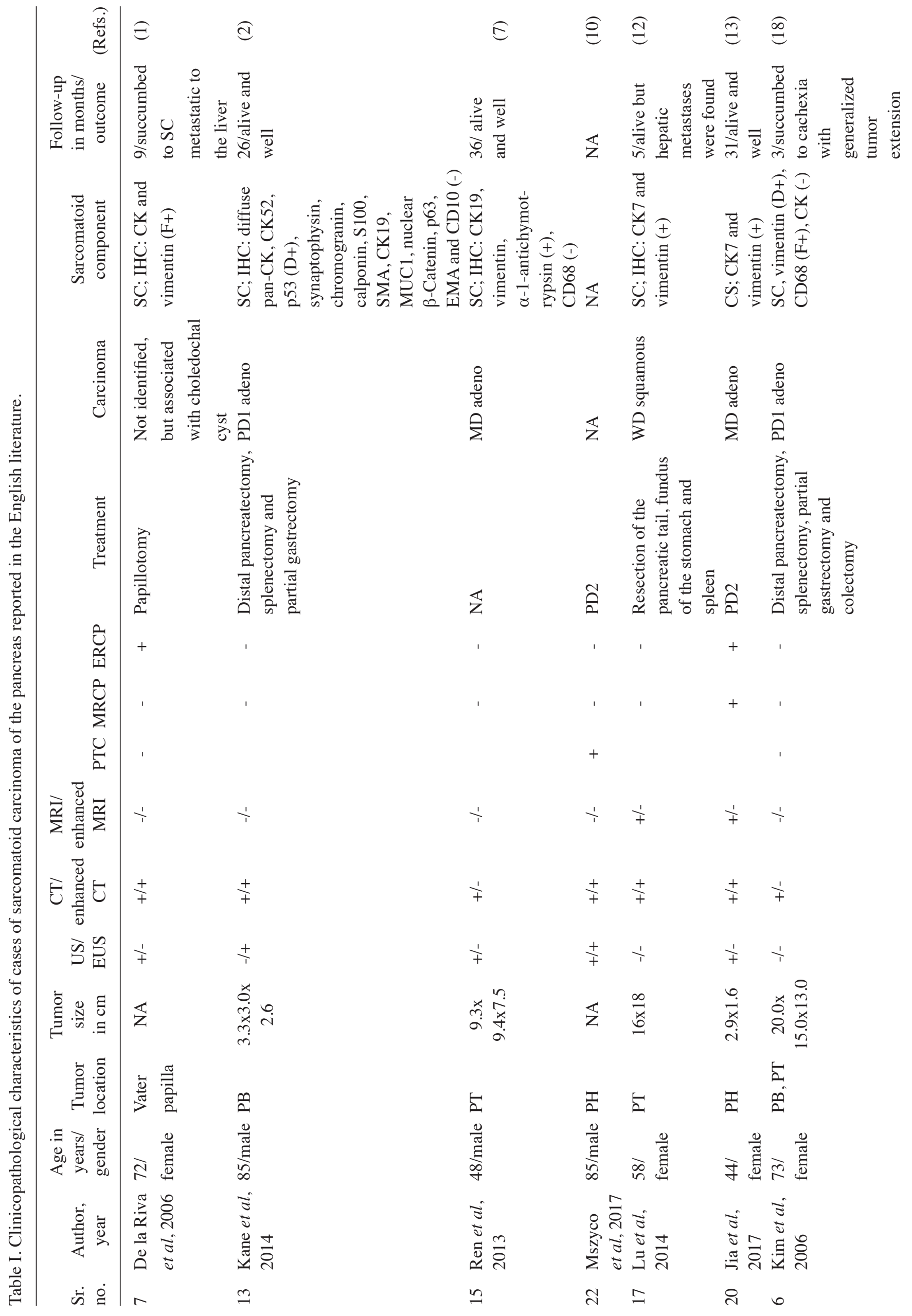




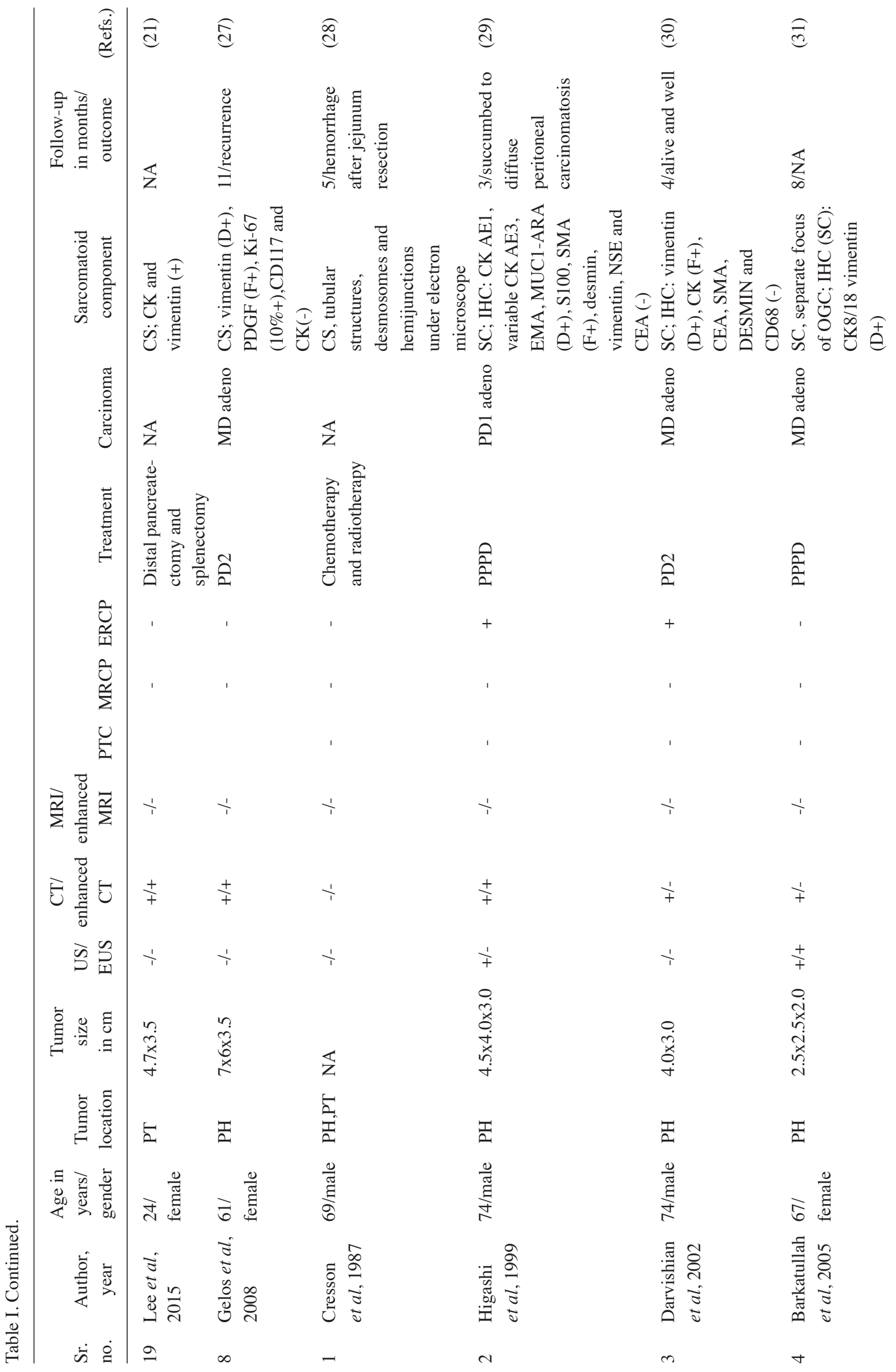




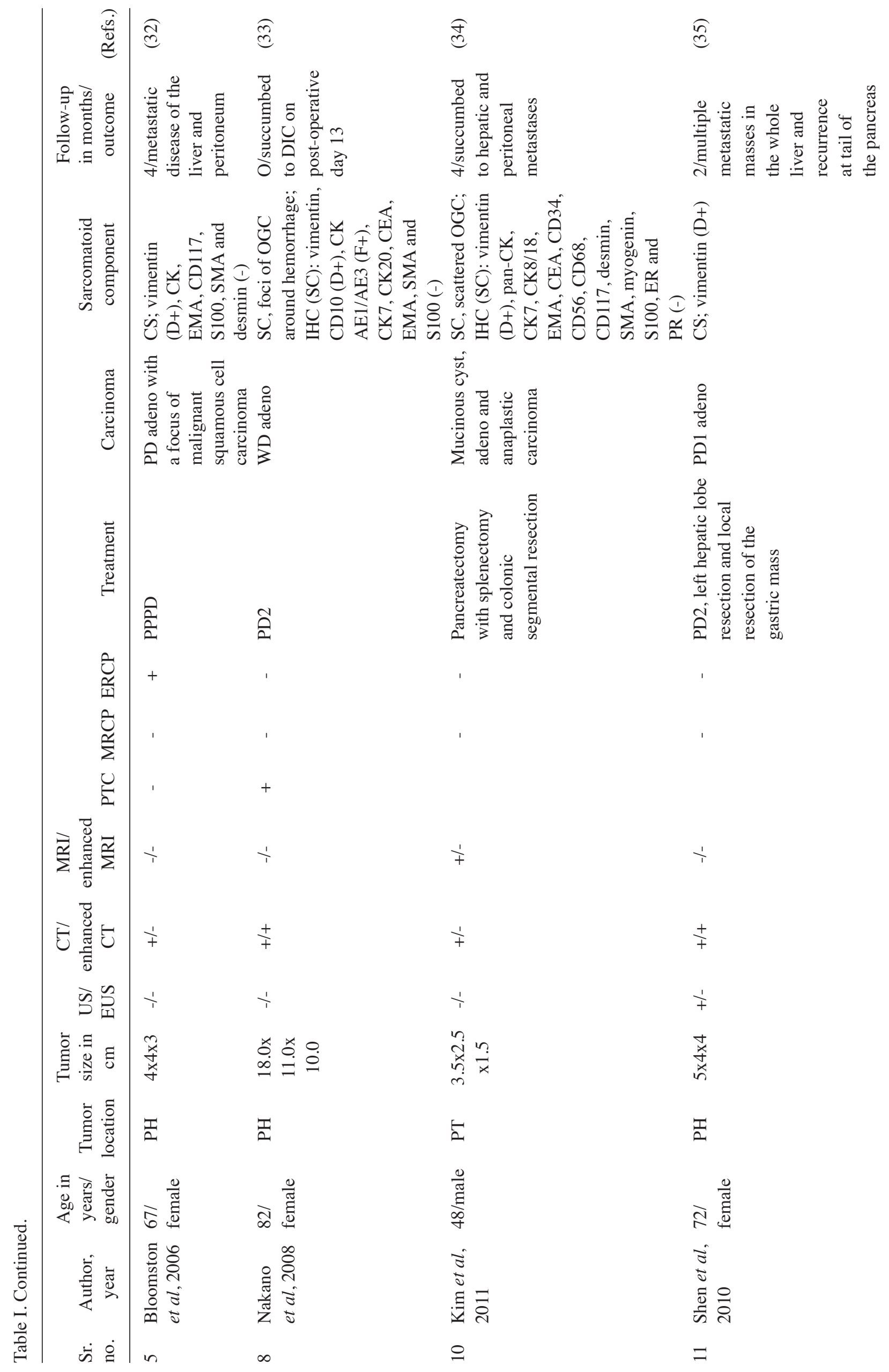




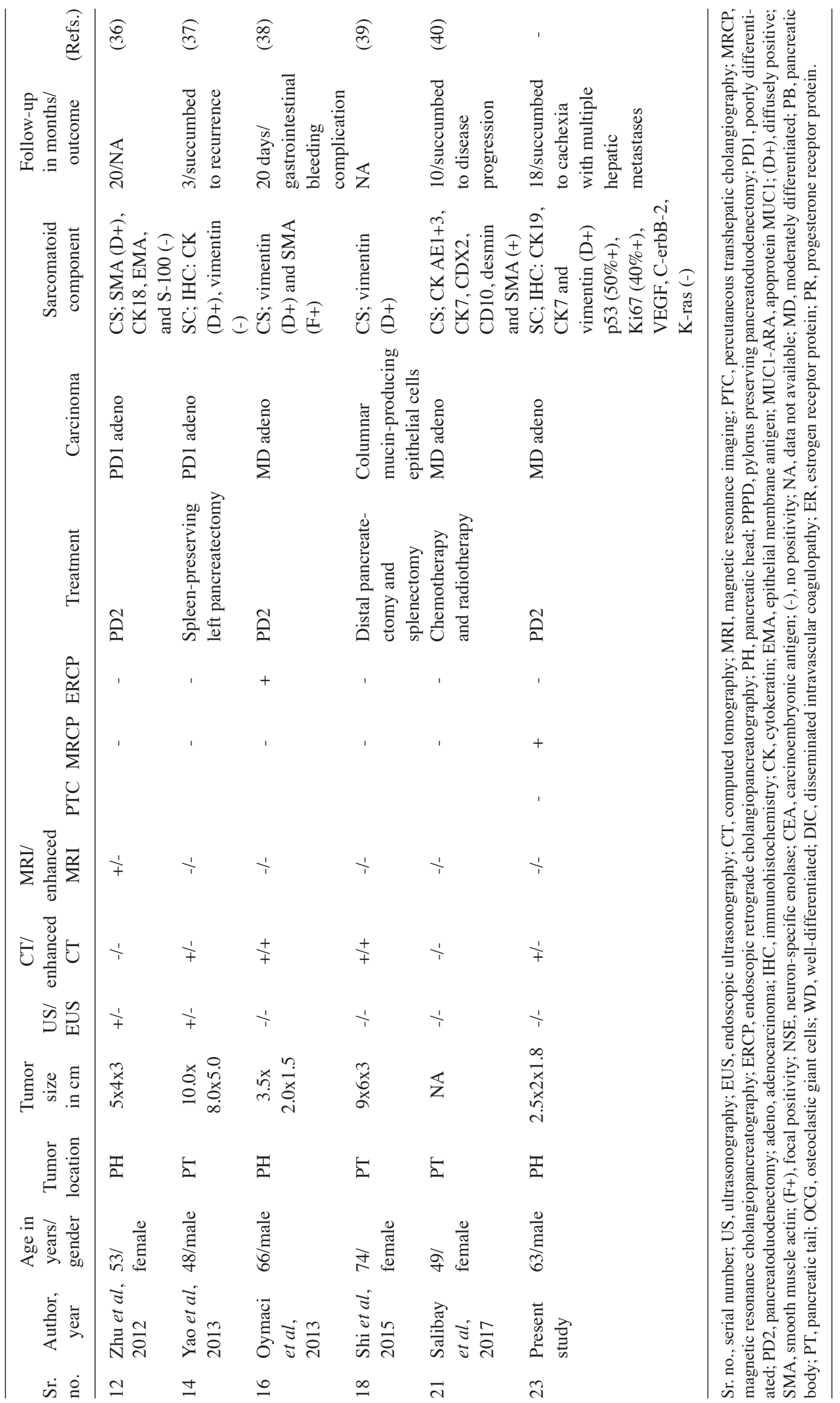


constituted the mesenchymal components. Ordinarily, the proportion of the sarcomatoid part should be greater than $50 \%$ to receive a diagnosis of SC (16). A study by Alguacil-Garcia classified SC into four histological subtypes based on light microscopy (4): (a) spindle cell carcinoma, (b) osteoclastic giant cell tumors, (c) pleomorphic giant cell carcinoma, and (d) round cell anaplastic carcinoma. Additionally, some SC specimens show sarcomatoid constituents in metastasized lymph nodes, which show the tumorous nature of the sarcomatoid region rather than reacting hyperplasia of the tissue.

Immunohistochemistry, electron microscopy and genetic studies are of great value in diagnosing SC. Generally, the mesenchymal components of SC express both mesenchymal and epithelial markers, which is critical for SC diagnosis. In the present case, the neoplasm primarily consisted of spindle cells with few epithelial components; however, expression of both mesenchymal and epithelial markers of the spindle cells supported a diagnosis of SC. Vimentin is the most common mesenchymal marker. Other myogenic markers (such as SMA, actin, desmin and myoglobin), neurogenic markers and osteogenic markers may also be positive in related components. Among epithelial markers, CK and EMA may be expressed in epithelial and/or sarcomatoid regions. Electron microscopy may show chitin mother cell granules, tonofibrils, desmosomes and melanin granules in SC spindle cells (17). Among genetic studies, p53 overexpression in both components of SC was found in a Korean case report (18). Furthermore, Almoguera found a KRAS mutation at codon 12 of exon 2 in spindle cells of two SCP cases (19). The identical genetic mutation of epithelial and mesenchymal components provides strong evidence for an EMT mechanism.

Radical resection of the neoplasm is the primary treatment for SCP (20). PD and pylorus-preserving PD (PPPD) are the major procedures for SCP of the pancreatic head, whereas SCP of the pancreatic body and tail is commonly treated with partial resection of the pancreas with splenectomy. Chemotherapy is necessary for cases with metastases in the lymph nodes (3). The prognosis of SCP is extremely poor (21-23) and may be associated with the tumor's volume and histological type, and the extent of local lymph node metastases. The average life expectancy is 2-3 months $(24,25)$, with a 3 -year survival rate of less than $3 \%$ (26). According to a report by Gelos, the average post-operative survival interval was 6 months and the longest living patient survived for 15 months (27). According to our statistics of SCP reported in English literature (Table I), the average post-operative survival of SCP is $10.15 \pm 10.23$ months. In this case, the patient lived for 18 months after surgery, which suggests that early, radical surgery extends survival for patients with SCP.

SCP is extremely rare, and its clinical presentation is similar to that of pancreatic ductal adenocarcinoma. Diagnosis is made on histopathological examination of the resected specimen. SCP has a poor prognosis, and is primarily treated with surgery; no specific adjuvant therapy is available.

\section{Acknowledgements}

The authors would like to thank Mrs Zhehui Wang for providing the pathological section and associated figure legends.

\section{Funding}

The present study was supported by grants from the Finance Department of Jilin Province (grant nos. SCZSYZ01502, SCZSYZ01521 and SCZSYZ01522).

\section{Availability of data and materials}

The datasets used during the present study are available from the corresponding author on reasonable request.

\section{Authors' contributions}

YJX and YX designed the study and wrote the manuscript. YY and XZ contributed to the manuscript revision. DZ, XY and JS contributed to the collection of clinical information and associated literature of SCP.

\section{Ethics approval and consent to participate}

The research was approved and consented by the Ethics Committee of the Second Hospital of Jilin University.

\section{Patient consent for publication}

The written consent to publish was obtained from the patient.

\section{Competing interests}

The authors declare that they have no competing interests.

\section{References}

1. De la Riva S, Muñoz-Navas MA, Betés M, Súbtil JC, Carretero C and Sola JJ: Sarcomatoid carcinoma of the pancreas and congenital choledochal cyst. Gastrointest Endosc 64: 1005-1006, 2006.

2. Kane JR, Laskin WB, Matkowskyj KA, Villa C and Yeldandi AV: Sarcomatoid (spindle cell) carcinoma of the pancreas: A case report and review of the literature. Oncol Lett 7: 245-249, 2014.

3. Fang XH: New arguments on carcinosarcoma (CS) and sarcomatoid carcinoma (SC). Cancer Res Clinic 17: 138-139, 2005 (In Chinese).

4. Alguacil-Garcia A and Weiland LH: The histologic spectrum, prognosis, and histogenesis of the sarcomatoid carcinoma of the pancreas. Cancer 39: 1181-1189, 1977.

5. Fukushima N, Hruban RH and Kato Y: Ductal adenocarcinoma variants and mixed neoplasms of the pancreas. WHO Classific Tumors Digest System: 292-299, 2010

6. Hruban RH, Pitman MB and Klimstra DS: Adenocarcinoma variants. In: Tumors of the Pancreas. AFIP Atlas of Tumor Pathology: Series 4. American Registry of Pathology, Washington, DC, pp165-190, 2007.

7. Ren CL, Jin P, Han CX, Xiao Q, Wang DR, Shi L, Wang DX and Chen H: Unusual early-stage pancreatic sarcomatoid carcinoma. World J Gastroenterol 19: 7820-7824, 2013.

8. Shirobe T, Yamamoto T and Mori T: A case of spindle cell carcinoma of the pancreas. Suizo 10: 387-392, 1995

9. Wolfman NT, Karstaedt N and Kawamoto EH: Pleomorphic carcinoma of the pancreas: Computed-tomographic, sonographic, and pathologic findings. Radiology 154: 329-332, 1985.

10. Mszyco S, Teng L, Annunziata J and Hartman MS: Pancreatic carcinosarcoma: A case report highlighting computed tomography characteristics. Curr Probl Diagn Radiol 46: 342-345, 2017.

11. Husain AN, Colby TV, Ordóñez NG, Krausz T, Borczuk A, Cagle PT, Chirieac LR, Churg A, Galateau-Salle F, Gibbs AR, et al: Guidelines for pathologic diagnosis of malignant mesothelioma: A consensus statement from the International Mesothelioma Interest Group. Arch Pathol Lab Med 133: 1317-1331, 2009. 
12. Lu BC, Wang C, Yu JH, Shen ZH and Yang JH: A huge adenosquamous carcinoma of the pancreas with sarcomatoid change: An unusual case report. World J Gastroenterol 20: 16381-16386, 2014.

13. Jia Z, Zhang K, Huang R, Zhou X and Jiang L: Pancreatic carcinosarcoma with rare long-term survival: Case report and review of the literature. Medicine (Baltimore) 96: e5966, 2017.

14. Yu W, Wang Y, Jiang Y, Zhang W and Li Y: Distinct immunophenotypes and prognostic factors in renal cell carcinoma with sarcomatoid differentiation: A systematic study of 19 immunohistochemical markers in 42 cases. BMC Cancer 17: 293, 2017.

15. Inoue $\mathrm{H}$, Takahashi $\mathrm{H}$, Hashimura M, Eshima K, Akiya M, Matsumoto $\mathrm{T}$ and Saegusa M: Cooperation of Sox 4 with $\beta$-catenin/p300 complex in transcriptional regulation of the Slug gene during divergent sarcomatous differentiation in uterine carcinosarcoma. BMC Cancer 16: 53, 2016.

16. Ding HY and Liao SL: Carcinosarcoma (CS) and sarcomatoid carcinoma (SC). Chin J Diagn Pathol 6: 56-57, 1999 (In Chinese).

17. Lichtiger B, Mackay B and Tessmer CF: Spindle-cell variant of squamous carcinoma. A light and electron microscopic study of 13 cases. Cancer 26: 1311-1320, 1970.

18. Kim KH, Kang DY, Lee MK, Yang HW and Han HY: Sarcomatoid carcinoma of the pancreas: A case report. Kor J Pathol 40: 306-310, 2006

19. Almoguera C, Shibata D, Forrester K, Martin J, Arnheim N and Perucho M: Most human carcinomas of the exocrine pancreas contain mutant c-K-ras genes. Cell 53: 549-554, 1988

20. Dall'Oglio MF, Lieberknecht M, Gouveia V, Sant'Anna AC, Leite KR and Srougi M: Sarcomatoid differentiation in renal cell carcinoma: Prognostic implications. Int Braz J Urol 31: 10-16, 2005.

21. Lee J, Hyun JJ and Lee HS: A Rare cause of abdominal pain by pancreatic mass in a young female patient. Carcinosarcoma of the pancreas. Gastroenterology 149: e3-e5, 2015.

22. Goto T, Hirotsu Y, Mochizuki H, Nakagomi T, Oyama T, Amemiya $\mathrm{K}$ and Omata M: Stepwise addition of genetic changes correlated with histological change from 'well-differentiated' to 'sarcomatoid' phenotypes: A case report. BMC Cancer 17: 65, 2017.

23. Schaefer IM, Sahlmann CO, Overbeck T, Schweyer S and Menke J: Blastomatoid pulmonary carcinosarcoma: Report of a case with a review of the literature. BMC Cancer 12: 424, 2012.

24. Kurihara K, Nagai H, Kasahara K, Kawai T, Saito K and Kanazawa K: Pleomorphic carcinoma of the pancreas with massive lymphocytic stromal infiltration and long-term survival after resection. Int J Pancreatol 27: 241-248, 2000.

25. Tschang TP, Garza-Garza R and Kissane JM: Pleomorphic carcinoma of the pancreas: An analysis of 15 cases. Cancer 39: 2114-2126, 1977.

26. Kamisawa T, Tabata I, Isawa T, Tsuruta K, Okamoto A and Koike M: A case of pleomorphic carcinoma of the pancreas showing sequential histological change by immunohistochemical study. Int J Pancreatol 18: 67-70, 1995.
27. Gelos M, Behringer D, Philippou S and Mann B: Pancreatic carcinosarcoma. Case report of multimodal therapy and review of the literature. JOP 9: 50-55, 2008

28. Cresson DH and Reddick RL: Sarcomatoid carcinoma of the pancreas presenting as gastric carcinoma: Clinicopathologic and ultrastructural findings. J Surg Oncol 36: 268-274, 1987.

29. Higashi M, Takao S and Sato E: Sarcomatoid carcinoma of the pancreas: A case report with immunohistochemical study. Pathol Int 49: 453-456, 1999.

30. Darvishian F, Sullivan J, Teichberg S and Basham K Carcinosarcoma of the pancreas: A case report and review of the literature. Arch Pathol Lab Med 126: 1114-1117, 2002.

31. Barkatullah SA, Deziel DJ, Jakate SM, Kluskens L and Komanduri S: Pancreatic carcinosarcoma with unique triphasic histological pattern. Pancreas 31: 291-292, 2005.

32. Bloomston M, Chanona-Vilchis J, Ellison EC, Ramirez NC and Frankel WL: Carcinosarcoma of the pancreas arising in a mucinous cystic neoplasm. Am Surg 72: 351-355, 2006.

33. Nakano T, Sonobe H, Usui T, Yamanaka K, Ishizuka T, Nishimura $E$ and Hanazaki K: Immunohistochemistry and K-ras sequence of pancreatic carcinosarcoma. Pathol Int 58: 672-677, 2008.

34. Kim HS, Joo SH, Yang DM, Lee SH, Choi SH and Lim SJ: Carcinosarcoma of the pancreas: A unique case with emphasis on metaplastic transformation and the presence of undifferentiated pleomorphic high-grade sarcoma. J Gastrointestin Liver Dis 20: 197-200, 2011.

35. Shen ZL, Wang S, Ye YJ, Wang YL, Sun KK, Yang XD and Jiang KW: Carcinosarcoma of pancreas with liver metastasis combined with gastrointestinal stromal tumour of the stomach: Is there a good prognosis with the complete resection? Eur J Cancer Care (Engl) 19: 118-123, 2010.

36. Zhu WY, Liu TG and Zhu H: Long-term recurrence-free survival in a patient with pancreatic carcinosarcoma: A case report with a literature review. Med Oncol 29: 140-143, 2012.

37. Yao J, Qian JJ, Zhu CR, Bai DS and Miao Y: Laparoscopic left pancreatectomy for pancreatic sarcomatoid carcinoma: A case report and review of the literature. Oncol Lett 6: 568-570, 2013.

38. Oymaci E, Argon A, Coşkun A, Uçar AD, Carti E, Erkan N and Yildirim M: Pancreatic carcinosarcoma: Case report of a rare type of pancreatic neoplasia. JOP 14: 212-215, 2013.

39. Shi HY, Xie J and Miao F: Pancreatic carcinosarcoma: First literature report on computed tomography imaging. World J Gastroenterol 21: 1357-1361, 2015.

40. Salibay CJ, Rewerska J, Gupta S and Ree N: Primary carcinosarcoma of the pancreas with CD10-positive sarcoma component. J Investig Med High Impact Case Rep 5: 2324709617740906 , 2017. 